\title{
Realization of the purely spatial Einstein-Podolsky-Rosen paradox in full-field images of spontaneous parametric down conversion.
}

\author{
Paul-Antoine Moreau, Joé Mougin-Sisini, Fabrice Devaux, and Eric Lantz \\ Institut FEMTO-ST Département d'Optique PM Duffieux \\ UMR CNRS - Université de Franche-Comté $n^{\circ} 6174$, \\ Route de Gray 25030 Besançon Cedex, FRANCE
}

(Dated: September 23, 2018)

\begin{abstract}
We demonstrate Einstein-Podolsky-Rosen (EPR) entanglement by detecting purely spatial quantum correla-tions in the near and far fields of spontaneous parametric down-conversion generated in a type- 2 beta barium borate crystal. Full-field imaging is performed in the photon-counting regime with an electron-multiplying CCD camera. The data are used without any postselection, and we obtain a violation of Heisenberg inequalities with inferred quantities taking into account all the biphoton pairs in both the near and far fields by integration on the entire two-dimensional transverse planes. This ensures a rigorous demonstration of the EPR paradox in its original positionmomentum form.

PACS numbers: 03.65.Ud, 42.50.Dv, 42.50.Ar, 42.50.Lc
\end{abstract}

\section{INTRODUCTION}

In 1935, Einstein, Podolsky, and Rosen (EPR) proposed a Gedankenexperiment [1] involving two spatially separated but entangled particles. They showed that quantum mechanics predicts that these particles could have both perfectly correlated positions and momenta, in contradiction with the so-called local realism where two distant particles should be treated as two different sys- tems. Though the original intention of EPR was to show that quantum mechanics is not complete, the standard present view is that entangled particles do experience nonlocal correlations [2, 3].

Recently, the realization and detection of entangled EPR states aroused much interest of the scientific community fol-lowing a testable formulation of the EPR paradox introduced by Reid [4], involving correlations of quadratures of twin beams. A recent review on the subject has been given in Ref. [5]. Furthermore, position and momentum entanglement of beams have been demonstrated at the EPR level by combin-ing squeezed light from two spatial modes, with measurements by homodyne detection in the temporal domain [6, 7].

Spontaneous parametric down-conversion (SPDC) provides independent pairs of entangled photons that makes the systemvery close to that considered in the original EPR paper: the positions of photons 1 and 2 are detected in the near field, and their momenta correspond to the far field. Howellet al. 8] havemeasured in both planes the probability distribution of the position of photon 2 , conditioned by the detection of photon 1 . The product of the conditional variances was found to be 25 times smaller than the limit for the product of variances for a single photon given by Heisenbergs uncertainty relation. This impressive result was obtained by measuring tempo-ral coincidences between cross-polarized photons in type-2 SPDC. These photons were separated by a polarizing beam splitter: for a fixed position of a narrow slit transmitting photon 1 to an avalanche photodiode, the level of coincidences was measured for each position of a similar slit transmitting photon 2 to a separate similar detector. Hence, in the words of Reid et al. [5], "detection events are only considered if two emitted photons are simultaneously detected". In this sense, they did not prospect the full EPR characteristic of SPDC but a monodimensional and point EPR paradox using postselected data. The same comment could be made on the recent realization by Leach et al. [9], where point detection was used in one of the transverse dimensions of the field.

We present here a full spatial demonstrationof EPRsteering by imaging highly spatially multimode type-2 SPDC with an electron-multiplying CCD (EMCCD) camera. This ensures taking into account each detection event that occurs in a relatively long exposure time compared to the laser pulse duration and, more importantly, compared to the coincidence time detection used in the experiments that use correlation data detection. Hence, even if there are losses and false-positive and -negative events, these spurious events are random, which is fundamentallydifferent fromconsideringapriorithat pairs are correlated and detecting only in the temporal and spatial gate where the twinphoton arrives. Second,Heisenberg inequalities concern a sole system, hence it is meaningless to test these inequalities if the near field and the far field do not correspond to this unique system. By treating full-field bidimensional images of photodetection and measuring variances in two orthogonal directions, we assure a perfect correspondence between the subsystems involved in the near field and in the far field [5], in contrast to point or one-dimensional (1D) detection. Moreover we demonstrate not only an EPR paradox in two spatial transverse dimensions but also one using an isotropic criterion involving the whole system. Note that the use of type- 2 phase matching allows us to spatially separate the idler and signal photons to be close to the real conditions of an EPR test of local realism. Detection is performed with an EMCCD camera. The ability 
of EMCCD cameras to reach the photon-counting regime with a very high quantum efficiency [10, 11] makes them useful in quantum optics, and they have already been used by our group to characterize quantum correlations in the far field of type-1 [12, 13] and type-2 [14] SPDC. Note that previous work has been done by other groups to detect the quantum correlation in the far field [15, 16], using intensified CCD (ICCD) cameras that have a lower quantum efficiency.

\section{THEORY}

SPDC induced by a wide monomode Gaussian pump is a strongly multimode beam: the extension of the downconverted beam in the near field (image plane) is identical to that of the pump in the limit of low gain and for a sufficiently wide and thin crystal, while the far-field (Fourier plane) extension is limited by phase matching. The etendue of the beam, i.e., the product of its transverse surface by the solid angle it subtends or the number of transverse modes in appropriate units, corresponds to the two-photon Schmidt number [17].

The spatial extension of a mode in either the near or the far field is proportional to the inverse of the full beam extension in the other plane. For single-photon imaging, the laws of diffraction are equivalent to Heisenbergs uncertainty relation: a photon that can be localized in one mode of the near field, for example, by traversing an aperture of the size corresponding to the mode, will be detected at a random position in the entire far-field diffraction pattern. However, the laws of quantum mechanics state that a pair of signal-idler photons will be detected either in the same mode in the near field or in opposite modes in the far field if no detection occurs in the other plane. Because the detection plane can be chosen at a time when causal interaction between photons is no longer possible, these correlations are not compatible with local realism, as demonstrated first in the EPR paper [1], though compatible with Heisenbergs uncertainty relation since correlations cannot be measured in both planes for the same photon pair.

We can describe the SPDC behavior as follows: for a detection of a photon 1 at $\mathbf{r}_{1}$, the probability density of detection of a photon 2 at $\mathbf{r}_{2}$ can be written as:

$$
p\left(\mathbf{r}_{\mathbf{2}} \mid \mathbf{r}_{\mathbf{1}}\right)=p\left(\mathbf{r}_{\mathbf{2}}\right)+f(\boldsymbol{\Delta} \mathbf{r})
$$

where $p\left(\mathbf{r}_{\mathbf{2}}\right)$ is the probability density of detecting a photon of another pair (accidental coincidences) and $f(\boldsymbol{\Delta} \mathbf{r})$ is the probability density of detection of the twin photon, with $\Delta \mathbf{r}=\left\|\mathbf{r}_{\mathbf{2}} \pm \mathbf{r}_{\mathbf{1}}\right\|$, plus sign indicating the far field (correlation of momenta on oppositemodes) and theminus sign the near field. Translational invariance, circular symmetry, and independence of the pairs (pure SPDC without further amplification) are assumed. Hence, if $\mathrm{N}_{1}$ is the number of photons 1 detected on a surface $S_{1}$ and
$\mathrm{N}_{2}$ the corresponding quantity for photons 2, we have:

$$
\begin{aligned}
\left\langle N_{1} N_{2}\right\rangle & =\int_{S_{1}} d^{2} \mathbf{r}_{\mathbf{1}} \int_{S_{2}} d^{2} \mathbf{r}_{\mathbf{2}} p\left(\mathbf{r}_{\mathbf{1}}, \mathbf{r}_{\mathbf{2}}\right) \\
& =\int_{S_{1}} d^{2} \mathbf{r}_{\mathbf{1}} \int_{S_{2}} d^{2} \mathbf{r}_{\mathbf{2}}\left\{p\left(\mathbf{r}_{\mathbf{1}}\right) p\left(\mathbf{r}_{\mathbf{2}}\right)+p\left(\mathbf{r}_{\mathbf{1}}\right) f(\boldsymbol{\Delta} \mathbf{r})\right\}
\end{aligned}
$$

Therefore, the probability of detection in $\mathrm{S}_{2}$ of the twin photon 2 of the photon 1 detected on $S_{1}$ is simply given by:

$$
F\left(S_{2}\right)=\int_{S_{2}} d^{2} \mathbf{r}_{2} f(\boldsymbol{\Delta} \mathbf{r})=\frac{\left\langle N_{1} N_{2}\right\rangle-\left\langle N_{1}\right\rangle\left\langle N_{2}\right\rangle}{\left\langle N_{1}\right\rangle}
$$

If $S_{1}$ and $S_{2}$ are the same size, this expression can be symmetrized and becomes the normalized intercorrelation function:

$$
F\left(S_{2}\right)=F\left(S_{1}\right)=\frac{\left\langle N_{1} N_{2}\right\rangle-\left\langle N_{1}\right\rangle\left\langle N_{2}\right\rangle}{\frac{1}{2}\left(\left\langle N_{1}\right\rangle+\left\langle N_{2}\right\rangle\right)}
$$

Themean in this equation can be estimated by spatial averages on the different pixels of the image for a fixed $\Delta \mathbf{r}$, given by the intercorrelation of two "regions of interest" (ROIs) of an image, each one corresponding to one polarization of the SPDC. We will therefore obtain a nonlocal estimation involving all the light. Note that deterministic spatial variations of the mean intensity do not preclude the validity of these spatial averages, inasmuch as the width of the intercorrelation function is smaller than the scale of this deterministic variation. Indeed, the covariance signal-idler for a region formed by independent area is the sum of the covariances of each area, just as the mean for the region is the sumof themeans for each area. Hence, if the ratio between the covariance and the mean intensity does not depend of this mean, it will be retrieved by spatial averaging even if the mean varies spatially. Because of the weak signal-to-noise ratio, we proceed to an additional statistical average on different images taken at different times for the same system configuration.

For independent pairs, the quantity in equation (4) can be expressed as a function of the variance of the difference between $\mathrm{N}_{1}$ and $\mathrm{N}_{2}$ :

$$
\begin{gathered}
\left\langle N_{1}\right\rangle=\left\langle N_{2}\right\rangle=\left\langle N_{1}^{2}\right\rangle-\left\langle N_{1}\right\rangle^{2} \\
\Rightarrow F\left(S_{2}\right)=1-\frac{\left\langle\left(N_{1}-N_{2}\right)^{2}\right\rangle}{\left\langle N_{1}+N_{2}\right\rangle}
\end{gathered}
$$

The physical quantities used to test the EPR violation of Heisenberg inequalities are the spatial variances in each dimension $\Delta^{2} x$ and $\Delta^{2} y$. To define a unique global criterion, we use the mean value of the two variances (i.e. half of the mean of the squared distance) :

$$
\Delta^{2} r, p=\frac{\Delta^{2} x, p_{x}+\Delta^{2} y, p_{y}}{2}
$$

Thus, by introducing the Heisenberg inequalities in the product of near-field and far-field variances, one gets

$$
\Delta^{2} r \Delta^{2} p=\frac{1}{4}\left(\Delta^{2} x+\Delta^{2} y\right)\left(\Delta^{2} p_{x}+\Delta^{2} p_{y}\right) \geq \frac{\hbar^{2}}{4}
$$


We have used the fact that $\Delta^{2} x \Delta^{2} p_{y}+\Delta^{2} y \Delta^{2} p_{x} \geq 2 \frac{\hbar^{2}}{4}$. Note that this two dimensional diffraction limit will only be reached by isotropic $2-\mathrm{D}$ gaussians.

\section{EXPERIMENT}

The experimental setup is represented in Fig. 1. The pump pulse at $355 \mathrm{~nm}$ provided by a passively Q-switched Nd:YAG laser (mean power: $27 \mathrm{~mW}$, pulse duration: $300 \mathrm{ps}$, repetition rate: $1 \mathrm{kHz}$ ), illuminates a 1-mm long type 2 BBO nonlinear crystal. The far-field image of the SPDC is formed on the EMCCD in the focal plane of a $37 \mathrm{~mm}$ focal aspheric lens: Fig. 1(a). In the near field configuration, Fig 1(b), the signal and idler photons are separated by a Wollaston prism of 1.5 of angular separation positioned around the Fourier plane. The plane in the middle of the BBO crystal is imaged on the EMCCD plane by a second identical aspheric lens, in order to minimize the walk-off effects [18]. The transversal magnification has been checked: $\gamma=1.003 \pm 0.005$. The back-illuminated EMCCD camera from Andor Technology (model iXon+ DU897-ECS-BV) has a quantum efficiency greater than $90 \%$ in the visible range. The detector area is formed by $512 \times 512$ pixels, with a pixel size of $s_{\text {pix }}=16 \times 16 \mu \mathrm{m}^{2}$ (i.e. $0.46 \times 0.46 \mathrm{mrad}^{2}$ in the far field after division by the focal length). We used a readout rate of $10 \mathrm{MHz}$ at 14 bits and the camera was cooled to $-85^{\circ} \mathrm{C}$. Measurements were performed for a crystal orientation corresponding to non critical phase matching at degeneracy, i.e. collinear orientation of the signal and idler Poynting vectors in the crystal [19]. Photon pairs emitted around the degeneracy are selected by mean of a narrow-band interferential filter centered at $710 \mathrm{~nm}$ $(\Delta \lambda=4 \mathrm{~nm})$. As in [13] and 14], the photon-counting regime is ensured by adjusting the exposure time in such a way that the mean fluence of SPDC was between 0.1 and 0.2 photon per pixel, in order to minimize the false detections [10]. Moreover, the use of pump pulses with 300 ps duration (much longer than the coherence time of SPDC) and an exposure time of the EMCCD of $10 \mathrm{~ms}$ (i.e. 10 laser shots) allow the excess noise to be limited by increasing the number of temporal modes [20] : the mean number of photons for one spatiotemporal mode is less than $10^{-3}$, in good agreement with the hypothesis of pure spontaneous parametric down conversion, without any stimulated amplification.

\section{A. Near field}

We show in Fig. 22 (a) the sum of the 10000 near field images. The two SPDC patterns are clearly visible, with inhomogeneities and hot spots due to defaults on the crystal. The ROIs corresponding to either the signal or the idler, large enough to encompass all the light for each polarization, have a common area. As a consequence, the intercorrelation function exhibits a strong
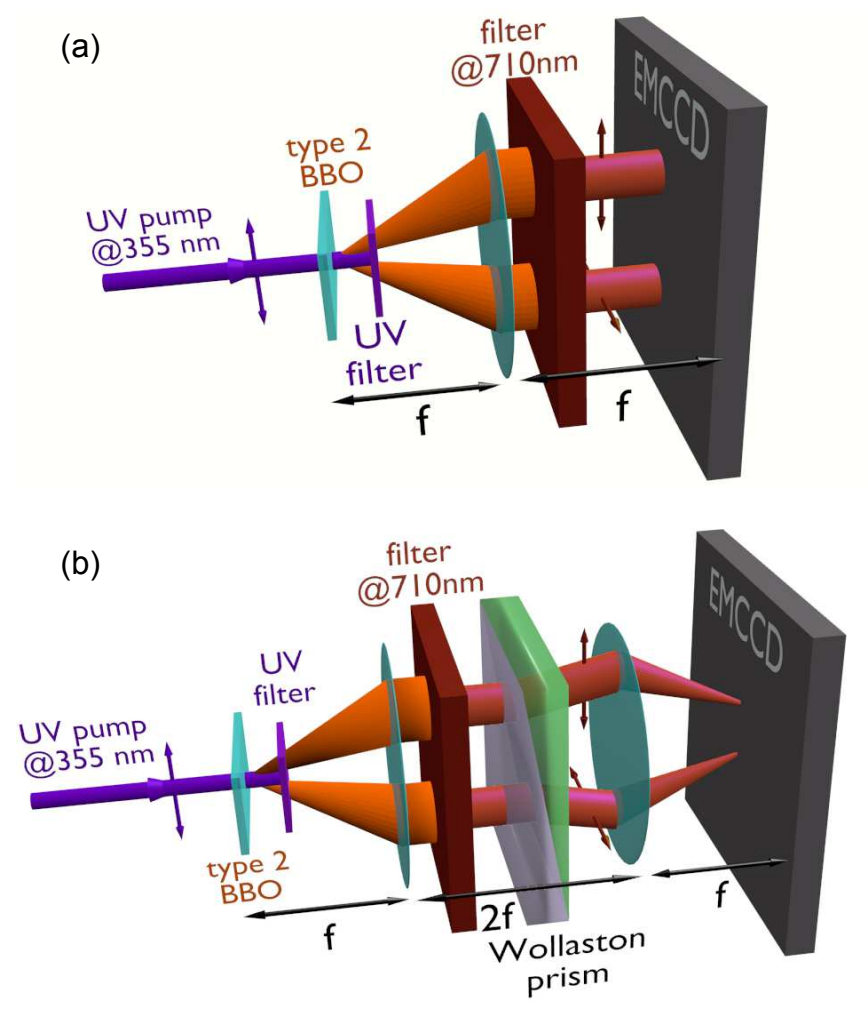

FIG. 1. Experimental setups. (a) Far field. (b) Near field. Brown arrows give the polarization directions
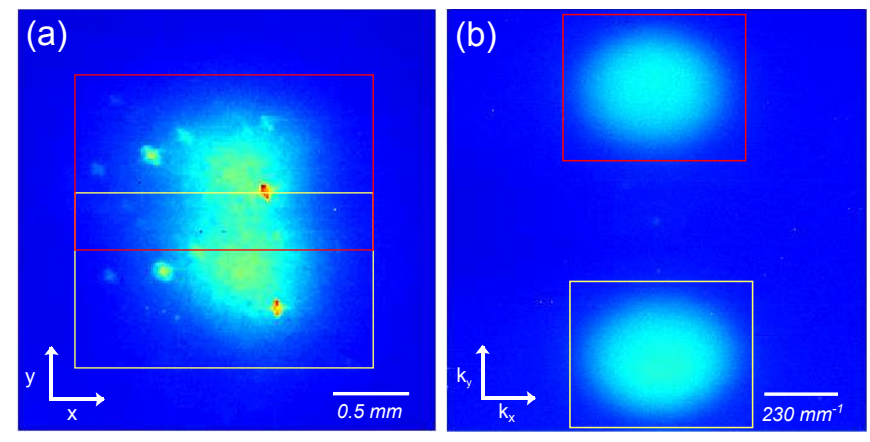

FIG. 2. Intensity distributions. (a) Near field. (b) Far field. In both figures rectangles denote the ROIs used for calculation.

autocorrelation peak, as can be seen in Fig. 3, but the intercorrelation peak, due to quantum correlations, and the autocorrelation peak are clearly distinguishable. We use a Fourier algorithm without any zero padding to compute the intercorrelation, which is equivalent to a periodisation of the images. Figure 4 presents the near-field intercorrelation peak ob-tained from ROIs taken in the same image and a witness intercorrelation obtained from ROIs taken in two successive images. The absence of any non-negligible intercorrelation value in the second image shows that the inhomogeneities in the crystal do not create any deterministic intercorrelation pat-tern. The first image exhibits a weak intercorrelation vertical line (more 


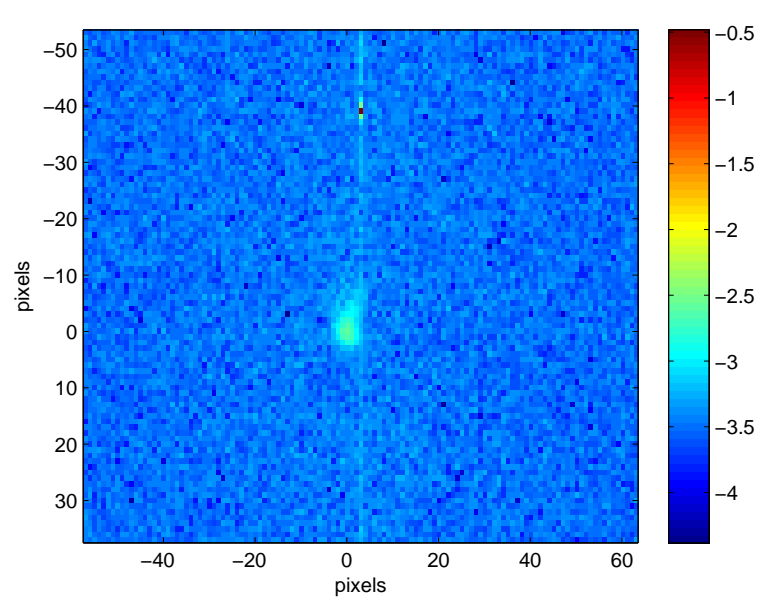

FIG. 3. Near-field complete intercorrelation in log scale.
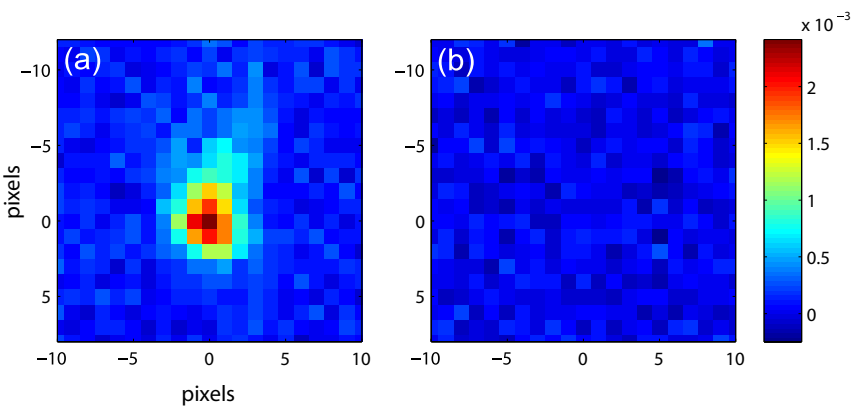

FIG. 4. (a) Near-field intercorrelation function. (b) Corresponding witness intercorrelation.

visible in 3 because of the log scale), which is due to the spreading of the autocorrelation peak induced by smearing in the gain register of the EMCCD camera. The existence of such an artifact again shows the importance of sufficiently separating the signal and idler photons on the camera to avoid any superposition of the autocorrelation and intercorrelation peaks. By fitting the intercorrelation peakwith a two-dimensional Gaussian function, we estimate the inferred near-field standard deviations in pixels:

$$
\Delta x=1.53 \pm 0.07, \Delta y=2.2 \pm 0.1, \Delta r=1.89 \pm 0.09
$$

By integrating thefitted curve,we alsoobtain the total quantum correlation coefficient in the near field, $R_{n}=$ $5 \times 10^{-2}$. This coefficient corresponds to the intercorrelation of pixels much larger than the coherence area [14].

\section{B. Far field}

Figure 2 (b) shows the sum of 10000 images in the far-field configuration. The intercorrelation function obtained in the far field is presented in Fig. 5. Note that the
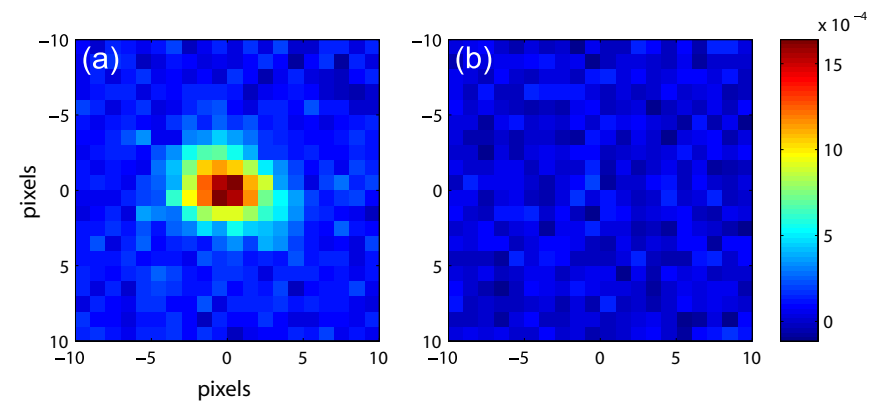

FIG. 5. (a) Far-field intercorrelation function. (b) Corresponding witness intercorrelation.

anisotropy of the peak is mainly due to the anisotropy of the shape of the pump. However, as predicted by simulations, an enlargement exists due to the imperfect degeneracy of the photons wavelength. This enlargement is itself anisotropic and, as predicted by the theory, is greater in the walk-off direction which separates the two fluorescence spots in the far field (vertical direction on each image presented here). The experimental results are in agreement with this phenomenon since the enlargement of the intercorrelation peak is greater in the vertical dimension than in the horizontal one 14]. We finally find the inferred standard deviations in far-field pixel units:

$\Delta p_{x}=2.35 \pm 0.08, \Delta p_{y}=1.85 \pm 0.07, \Delta p=2.11 \pm 0.07$

The total quantum correlation coefficient in the far field is $R_{p}=4.4 \times 10^{-2}$. In agreement with Eq. 5, we have experimentally shown in [14] that $\left(1-R_{p}\right)$ is equal to the variance of the difference between areas greater than the coherence cell, expressed in shot noise units.

\section{EPR VIOLATION OF THE HEISENBERG INEQUALITIES}

We are now able to test the violation of the Heisenberg inequalities by using the inferred quantitieswe havemeasured. In the horizontal direction, we have:

$$
\begin{aligned}
\Delta^{2} x \Delta^{2} p_{x} & =\left(1.53 \times 2.35 \frac{2 \pi \cdot s_{\text {pix }}}{f \cdot \lambda} \hbar\right)^{2} \\
& =(0.048 \pm 0.008) \hbar^{2}<\frac{\hbar^{2}}{4}
\end{aligned}
$$

giving a violation factor of $5.2 \pm 0.8$.

In the vertical direction, we find:

$$
\Delta^{2} y \Delta^{2} p_{y}=(0.06 \pm 0.01) \hbar^{2}<\frac{\hbar^{2}}{4}
$$

giving a violation factor of $4 \pm 1$.

And finally, by using the complete statistic of the fluorescence, from (6) and (7) one obtain:

$$
\Delta^{2} r \Delta^{2} p=(0.06 \pm 0.01) \hbar^{2}<\frac{\hbar^{2}}{4}
$$


which gives a violation factor of $4 \pm 1$.

\section{CONCLUSION}

We have demonstrated a purely spatial EPR paradox by using a full-field and direct detection method. The violation of the Heisenberg inequalities for the inferred quantities has been demonstrated for the whole system involving the two spatial transverse dimensions. By recording all the photon pairs generated by spontaneous parametric down-conversion in the near field and the far field of the same system, we make fewer supplementary assumptions than previous papers that involve postselection or homodyne detection. Hence, this demonstration of one of the most fascinating phenomena of quantum mechanics is made in the form closest to its original formulation.

\section{ACKNOWLEDGMENTS}

Some of the computations have been performed on the supercomputer facilities of the Mésocentre de calcul de Franche-Comté.
[1] A. Einstein, B. Podolsky, and N. Rosen, Phys. Rev. 47, 777 (1935).

[2] J. S. Bell, Physics 1, 195 (1964).

[3] A. Aspect, P. Grangier, and G. Roger, Phys. Rev. Lett. 47, 460 (1981).

[4] M. D. Reid, Phys. Rev. A 40, 913 (1989).

[5] M. D. Reid, P. D. Drummond, W. P. Bowen, E. G. Cavalcanti, P. K. Lam, H. A. Bachor, U. L. Andersen, and G. Leuchs, Rev. Mod. Phys. 81, 1727 (2009).

[6] K. Wagner, J. Janousek, V. Delaubert, H. Zou, C. Harb, N. Treps, J. F. Morizur, P. K. Lam, and H. A. Bachor, Science 321, 541 (2008)

[7] V. Boyer, A. M. Marino, R. C. Pooser, and P. D. Lett, Science 321, 544 (2008)

[8] J. C. Howell, R. S. Bennink, S. J. Bentley, and R. W. Boyd, Phys. Rev. Lett. 92, 210403 (2004)

[9] J. Leach, R. E. Warburton, D. G. Ireland, F. Izdebski, S. M. Barnett, A. M. Yao, G. S. Buller, and M. J. Padgett, Phys. Rev. A 85, 013827 (2012).

[10] E. Lantz, J.-L. Blanchet, L. Furfaro, and F. Devaux, Monthly Notices Of The Royal Astronomical Society 386, 2262 (2008).
[11] O. Jedrkiewicz, J.-L. Blanchet, E. Lantz, and P. D. Trapani, Optics Communications 285, 218 (2012)

[12] J.-L. Blanchet, F. Devaux, L. Furfaro, and E. Lantz, Phys. Rev. Lett. 101, 233604 (2008)

[13] J.-L. Blanchet, F. Devaux, L. Furfaro, and E. Lantz, Phys. Rev. A 81, 043825 (2010).

[14] F. Devaux, J. Mougin-Sisini, P.-A. Moreau, and E. Lantz, (to appear in EPJD). (2012).

[15] B. Jost, A. Sergienko, A. Abouraddy, B. Saleh, and M. Teich, Opt. Express 3, 81 (1998).

[16] S. S. R. Oemrawsingh, W. J. van Drunen, E. R. Eliel, and J. P. Woerdman, J. Opt. Soc. Am. B 19, 2391 (2002)

[17] M. P. van Exter, A. Aiello, S. S. R. Oemrawsingh, G. Nienhuis, and J. P. Woerdman, Phys. Rev. A 74, 012309 (2006).

[18] E. Brambilla, A. Gatti, M. Bache, and L. A. Lugiato, Phys. Rev. A 69, 023802 (2004).

[19] E. Lantz and F. Devaux, J. Opt. A 2, 362 (2000).

[20] E. Brambilla, L. Caspani, O. Jedrkiewicz, L. A. Lugiato, and A. Gatti, Phys. Rev. A 77, 053807 (2008) 